\title{
Observables in elastic electron-deuteron scattering with two-photon exchange
}

\author{
Yu Bing Dong ${ }^{1,2}$, Chung Wen Kao ${ }^{3}$, Shin Nan Yang ${ }^{4}$, and Yu Chun Chen ${ }^{4}$ \\ ${ }^{1}$ CCAST (World Laboratory), 100080 Beijing, P. R. China \\ ${ }^{2}$ Institute of High Energy Physics, Chinese Academy of Sciences, Beijing, P. R. China \\ ${ }^{3}$ Department of Physics, Chung-Yuan Christian University, Chung-Li 320, Taiwan \\ ${ }^{4}$ Department of Physics and Center for Theoretical Sciences, National Taiwan University, \\ Taipei 10617, Taiwan
}

July 1,2021

\begin{abstract}
The general form of the cross section as well as the polarizations of electron-deuteron elastic scattering are given. Two-photon exchange effects are analyzed. Possible signatures of the two-photon exchange effects in the electron-deuteron elastic scattering are discussed.
\end{abstract}

PACS: 13.40.GP, 25.30.-c, 25.30.Bf, 24.70.+s, 13.60.Fz

Keywords: Electron-deuteron scattering, Polarizations, One-photon-exchange, Two-Photon exchange. 


\section{Introduction}

Electromagnetic form factors of the nucleon provide us with essential information about the internal structure of the nucleon as they describe the distribution of charge and magnetization inside the nucleons. Traditionally, the Sachs electric $G_{E}\left(Q^{2}\right)$ and magnetic $G_{M}\left(Q^{2}\right)$ form factors are determined from the $e N$ elastic scattering by the Rosenbluth separation method. Namely, in the one-photon exchange (OPE) approximation, the unpolarized $e N$ cross section is a linear function of photon polarization parameter $\epsilon$ for a given value of four-momentum transfer squared $Q^{2}$,

$$
d \sigma_{0}=A_{0}\left(G_{M}^{2}\left(Q^{2}\right)+\frac{\epsilon}{\tau_{N}} G_{E}^{2}\left(Q^{2}\right)\right),
$$

where the factor $A_{0}$ depends on kinematic variables, and $\tau_{N}=Q^{2} / 4 M_{N}^{2}$ with $M_{N}$ the nucleon mass. $G_{M}\left(Q^{2}\right)$ and $G_{E}\left(Q^{2}\right)$ can then be determined from the intercept and the slope from the linear plot of $d \sigma_{0} v s . \epsilon$. The ratio $R=\mu_{p} G_{E} / G_{M}$ determined by the Rosenbluth separation method from the ep scattering, where $\mu_{p}=2.79$ is the proton magnetic moment, has been consistent with $R \approx 1$ for $Q^{2}<6 \mathrm{GeV}^{2}[1]$.

Another way to measure $R$ is with the use of polarized transfer technique. In the Born approximation for the elastic ep scattering, a longitudinally polarized electron transfers its polarization to the recoil proton with two nonzero components, $P_{t}$, perpendicular to, and $P_{l}$ parallel to, the proton momentum in the scattering plane. Simultaneous measurements of these two polarizations give [2]

$$
\frac{P_{t}}{P_{l}}=-\sqrt{\frac{2 \epsilon}{\tau_{N}(1+\epsilon)}} \frac{G_{E}}{G_{M}} .
$$

Recent polarization transfer experiments at Jefferson Lab [3, 4] give, however, a result $R \simeq 1-0.135\left(Q^{2}-0.24\right)$, which differs substantially from $R \approx 1$ as obtained by the Rosenbluth separation method over the same range of $Q^{2}[1$, exhibiting a nonscaling behavior.

Two-photon exchange effects have been proposed to account for the discrepancies between the electromagnetic form factors measured through the Rosenbluth separation method and the polarization transfer method [5, 6, 7]. In Ref. [8], the two-photon exchange contributions to the neutron electromagnetic form factors have been estimated and found to be important at large $Q^{2}$. It is hence natural to expect that the two-photon exchange mechanisms could also give a non-negligible contribution to the electron-deuteron elastic and quasi-elastic 
scattering. The question of the two-photon exchange (TPE) effects in the electron-deuteron $(e D)$ elastic scattering has been studied in Refs. [9, 10, 11, 12, 13, focusing on the possible signatures in the elastic cross sections. It is possible that the TPE effects in the $e D$ scattering might be more easily accessible in the polarization observables, just as in the case of ep scattering [14, 15]. It is hence important to go beyond the Born approximation and derive the expressions for all observables, including the polarizations, in the elastic electron-deuteron scattering to provide a theoretical framework for analyzing data and the extraction of the deuteron form factors beyond the OPE approximation in a model-independent way. These general expressions can also be useful in choosing certain kinematical regions and combinations of observables which would be more sensitive to the TPE effects.

In this article, we present a general formulation of the elastic electron-deuteron scattering with multiple photon exchanges and derive the formulas for all possible observables, including unpolarized differential cross section, three vector polarization observables $P_{x}, P_{y}$ and $P_{z}$ and three tensor polarization observables $T_{20}, T_{21}$ and $T_{22}$. These formulas are given in Sec. 2 . In Sec. 3, we examine the expressions obtained in Sec. 2 and explore the possibilities where the TPE effects might most likely be observed and summarize in Sec. 4.

\section{Electron-deuteron elastic scattering with multi-photon ex- change}

The electromagnetic form factors of the deuteron are defined by the matrix elements of the electromagnetic current $J_{\mu}(x)$ according to

$$
\begin{aligned}
<p_{4}, \lambda^{\prime}\left|J_{\mu}(0)\right| p_{2}, \lambda>= & -e_{D}\left\{\left[G_{1}\left(Q^{2}\right) \xi^{\prime *}\left(\lambda^{\prime}\right) \cdot \xi(\lambda)-G_{3}\left(Q^{2}\right) \frac{\left(\xi^{* *}\left(\lambda^{\prime}\right) \cdot q\right)(\xi(\lambda) \cdot q)}{2 M_{D}^{2}}\right] \cdot P_{\mu}\right. \\
& \left.+G_{2}\left(Q^{2}\right)\left[\xi_{\mu}(\lambda)\left(\xi^{\prime *}\left(\lambda^{\prime}\right) \cdot q\right)-\xi_{\mu}^{\prime *}\left(\lambda^{\prime}\right)(\xi(\lambda) \cdot q)\right]\right\}
\end{aligned}
$$

where $p_{4}, \xi^{\prime}, \lambda^{\prime}$ and $p_{2}, \xi, \lambda$ denote the momentum, helicity, and polarization vector of the final and initial deuterons, respectively. $q=p_{4}-p_{2}$ is the photon momentum, $P=p_{2}+p_{4}, Q^{2}=$ $-q^{2}$ the four-momentum transfer squared, $M_{D}$ the deuteron mass and $e_{D}$ is the charge of the deuteron. In the one-photon exchange approximation or Born approximation, the unpolarized differential cross section of the elastic electron-deuteron scattering

$$
e\left(p_{1}, s_{1}\right)+D\left(p_{2}, \xi\right) \rightarrow e\left(p_{3}, s_{3}\right)+D\left(p_{4}, \xi^{\prime}\right)
$$


in the laboratory frame is given by [16]

$$
\frac{d \sigma}{d \Omega}=\left.\frac{d \sigma}{d \Omega}\right|_{M o t t} I_{0}(O P E)
$$

with

$$
I_{0}(O P E)=A\left(Q^{2}\right)+B\left(Q^{2}\right) \tan ^{2} \frac{\theta}{2},
$$

where $\theta$ is the scattering angle of the electron, $(d \sigma / d \Omega)_{M o t t}$ is the Mott cross section for a structure-less particle

$$
\left.\frac{d \sigma}{d \Omega}\right|_{M o t t}=\left(\frac{\alpha}{2 E}\right)^{2} \frac{\cos ^{2} \frac{\theta}{2}}{\sin ^{4} \frac{\theta}{2}} \frac{1}{1+\frac{2 E}{M_{D}} \sin ^{2} \frac{\theta}{2}}=\sigma_{0} \cot ^{2} \frac{\theta}{2},
$$

and

$$
A\left(Q^{2}\right)=G_{c}^{2}\left(Q^{2}\right)+\frac{2}{3} \tau G_{M}^{2}\left(Q^{2}\right)+\frac{8}{9} \tau^{2} G_{Q}^{2}\left(Q^{2}\right), \quad B\left(Q^{2}\right)=\frac{4}{3} \tau(1+\tau) G_{M}^{2}\left(Q^{2}\right) .
$$

In Eqs. (5] 180$), \alpha$ is the fine structure constant, $\tau=Q^{2} / 4 M_{D}^{2}$, and $E$ is the incident electron energy. $G_{M}, G_{C}$ and $G_{Q}$ are, respectively, the deuteron magnetic, charge and quadrupole form factors. The relations between $G_{M}, G_{C}$ and $G_{Q}$ and the form factors $G_{1}, G_{2}$ and $G_{3}$ defined in Eq. (3) are

$$
G_{M}=G_{2}, \quad G_{Q}=G_{1}-G_{2}+(1+\tau) G_{3}, \quad G_{C}=G_{1}+\frac{2}{3} \tau G_{Q}
$$

The normalizations of the three form factors are, respectively, $G_{c}(0)=1, G_{Q}(0)=M_{D}^{2} Q_{D}=$ 25.83 , and $G_{M}(0)=1.714$.

Note that in the well-known Rosenbluth separation of Eq. (6), there are two unpolarized structure functions $A$ and $B$, and three independent form factors $G_{C}, G_{Q}$ and $G_{M}$, for the deuteron, a spin-one particle. To determine the three form factors completely, one thus needs at least, one polarization observable. The optimal choice, in the literature, is the polarization $T_{20}$ (or $P_{z z}$ ). This is because three tensor polarizations, like $T_{20}, T_{21}$ and $T_{22}$, can be measured in the $e D$ scattering with unpolarized beam and target while the observables like $T_{21}$ and $T_{22}$ are small in magnitude because they are proportional to $G_{M}$.

It is known that Lorentz, parity, charge-conjugation invariance dictate that the T-matrix for the elastic electron-nucleon can be expanded in terms of six independent Lorentz structures. In the limit of zero electron mass, helicity conservation would reduce them to three [6]. 
The remaining three structure functions are all complex. In the OPE approximation, these three complex form factors further simplify to two real form factors $G_{M}\left(Q^{2}\right)$ and $G_{E}\left(Q^{2}\right)$. In the case of electron-deuteron elastic scattering, there are 36 Lorentz invariant amplitudes which reduce to 18 if parity is conserved. With charge-conjugation invariance, only 9 of them are independent. In the limit of zero electron mass, i.e., helicity is conserved, then one ends up with only 6 independent amplitudes. In analogy to virtual Compton scattering (virtual photon $\rightarrow$ deuteron, proton $\rightarrow$ electron), we can express the most general form of the $e D$ elastic scattering as [17

$$
\mathcal{M}^{e D}=-e^{2} \bar{u}\left(p_{3}, s_{3}\right) \gamma_{\mu} u\left(p_{1}, s_{1}\right) \frac{1}{q^{2}} \sum_{i=1}^{6} \tilde{G}_{i} M_{i}^{\mu}
$$

where

$$
\begin{aligned}
M_{1}^{\mu} & =\left(\xi^{*} \cdot \xi\right) P^{\mu}, \\
M_{2}^{\mu} & =\left[\xi^{\mu}\left(\xi^{\prime *} \cdot q\right)-(\xi \cdot q) \xi^{* \mu}\right], \\
M_{3}^{\mu} & =-\frac{1}{2 M_{D}^{2}}(\xi \cdot q)\left(\xi^{\prime *} \cdot q\right) P^{\mu}, \\
M_{4}^{\mu} & =\frac{1}{2 M_{D}^{2}}(\xi \cdot K)\left(\xi^{*} \cdot K\right) P^{\mu}, \\
M_{5}^{\mu} & =\left[\xi^{\mu}\left(\xi^{\prime *} \cdot K\right)+(\xi \cdot K) \xi^{* \mu}\right], \\
M_{6}^{\mu} & =\frac{1}{2 M_{D}^{2}}\left[(\xi \cdot q)\left(\xi^{*} \cdot K\right)-(\xi \cdot K)\left(\xi^{* *} \cdot q\right)\right] P^{\mu} .
\end{aligned}
$$

with $K=p_{1}+p_{3}$. Generally speaking, the form factors $\tilde{G}_{i}$ with $i=1,6$, are complex functions of $s=\left(p_{1}+p_{2}\right)^{2}$ and $Q^{2}=-\left(p_{1}-p_{3}\right)^{2}$. They can be expressed as

$$
\tilde{G}_{i}\left(s, Q^{2}\right)=G_{i}\left(Q^{2}\right)+G_{i}^{(2)}\left(s, Q^{2}\right),
$$

where $G_{i}$ 's correspond to the contributions arising from the one-photon exchange and $G_{i}^{(2)}$, s stand for the rest which would come mostly from the TPE. In the OPE approximation, $G_{4}=G_{5}=G_{6}=0$. It is easy to see that $G_{i}(i=1,2,3)$ is of order of $(\alpha)^{0}$ and $G_{i}^{(2)}$ $(i=1, \ldots 6)$ are of order $\alpha$.

It is now straightforward though tedious to derive the unpolarized differential cross section for the $e D$ elastic scattering with the general form of the scattering amplitude of Eqs. (10-11) which includes the TPE effects. If the contributions from pure TPE are neglected and only the interference terms between the OPE and TPE contribution are retained, the resulting 
differential cross section can be expressed as

$$
\begin{aligned}
\frac{d \sigma}{d \Omega} & =\left.\frac{d \sigma}{d \Omega}\right|_{\text {Mott }} I_{0} \\
& =\left.\frac{d \sigma}{d \Omega}\right|_{\text {Mott }}\left\{\left[(A+\Delta A)+(B+\Delta B) \tan ^{2} \frac{\theta}{2}\right]+\Delta \sigma\left(\theta, Q^{2}\right)\right\} \\
& =\sigma_{0}\left\{\left[(A+\Delta A) \cot ^{2} \frac{\theta}{2}+(B+\Delta B)\right]+\Delta \sigma\left(\theta, Q^{2}\right) \cot ^{2} \frac{\theta}{2}\right\},
\end{aligned}
$$

where $A$ and $B$ are the same as the ones in Eq. (8). In Eq. (13), $\Delta$ is used to indicate the contributions coming from the interference terms between the OPE and TPE which have not been considered before [18, 19, 20, 21]. $\Delta A$ and $\Delta B$ are given as

$$
\begin{aligned}
\Delta A= & 2\left[G_{c} \operatorname{Re}\left(G_{C}^{(2) *}\right)+\frac{2}{3} \tau G_{M} \operatorname{Re}\left(G_{M}^{(2) *}\right)+\frac{8}{9} \tau^{2} G_{Q} \operatorname{Re}\left(G_{Q}^{(2) *}\right)\right] \\
& +\frac{4 \tau^{2}}{3}\left[(2 \tau+1) G_{1}-2(\tau+1) G_{2}+2 \tau(\tau+1) G_{3}\right] \operatorname{Re}\left(G_{4}^{(2) *}\right), \\
\Delta B= & \frac{8}{3} \tau(1+\tau) G_{M} \operatorname{Re}\left(G_{M}^{(2) *}\right),
\end{aligned}
$$

and

$$
\begin{aligned}
\Delta \sigma\left(\theta, Q^{2}\right)= & \frac{2}{3}\left\{2 \tau \cot ^{2} \frac{\theta}{2}\left[(2 \tau-1) G_{1}-2 \tau G_{2}+2 \tau^{2} G_{3}\right] \operatorname{Re}\left(G_{4}^{(2) *}\right)\right. \\
& +\frac{K_{0}}{M_{D}}\left[\left((2 \tau-1) G_{1}-2 \tau G_{2}+2 \tau^{2} G_{3}-2 \tau \tan ^{2} \frac{\theta}{2} G_{2}\right) \operatorname{Re}\left(G_{5}^{(2) *}\right)\right. \\
& \left.\left.+2 \tau\left((2 \tau+1) G_{1}-(2 \tau+1) G_{2}+2 \tau(\tau+1) G_{3}\right) \operatorname{Re}\left(G_{6}^{(2) *}\right)\right]\right\},
\end{aligned}
$$

where

$$
K_{0}^{2}=\left(p_{10}+p_{30}\right)^{2}=4 M_{D}^{2} \tau\left[(1+\tau)+\cot ^{2} \frac{\theta}{2}\right] .
$$

Note that $\Delta A$ and $\Delta B$ contain no explicit $\theta$-dependence, if all of $G_{i}^{(2)}, i=1-6$ would be independent of $\theta$, while the last term in Eq.(13) contains explicit $\theta$-dependence through $\Delta \sigma$ besides the factor $\cot ^{2}(\theta / 2)$. However, according to Eq. (12),$G_{i}^{(2)}$ are functions of $\theta$ and $Q^{2}$, because $s=s\left(\theta, Q^{2}\right)$ in the laboratory frame. Therefore $\Delta A$ and $\Delta B$ both depend on the $\theta$ through $G_{i}^{(2)}$.

We next turn to the polarization observables which enter in the $e D$ elastic scattering. The relations between the notation of Arnold et al. [18] and the popular one of Garcon and Orden [19] are

$$
\begin{gathered}
P_{z z}=\sqrt{2} \operatorname{Re}\left(T_{20}\right), \quad P_{x z}=-\sqrt{3} \operatorname{Re}\left(T_{21}\right), \quad\left(P_{x x}-P_{y y}\right)=2 \sqrt{3} \operatorname{Re}\left(T_{22}\right), \\
P_{z}=-\sqrt{\frac{2}{3}} \operatorname{Re}\left(T_{10}\right), \quad P_{y}=-\frac{2 \sqrt{3}}{3} \operatorname{Im}\left(T_{11}\right), \quad P_{x}=-\frac{2 \sqrt{3}}{3} \operatorname{Re}\left(T_{11}\right) .
\end{gathered}
$$


The tensor polarizations in the $e D$ unpolarized elastic scattering are $T_{20}\left(P_{z z}\right), T_{21}\left(P_{x z}\right)$, and $T_{22}\left(P_{x x}-P_{y y}\right)$ (the convention of Ref. [18]). In this paper, we use the notation of Ref. [18. There are several ways to extract those polarization observables. For example, the vector polarizations $P_{x}$ and $P_{z}$ can be measured by using the longitudinal polarized electron beam and the unpolarized deuteron target [18]. They are given by the the asymmetry of the cross section with the different polarizations of the electron beam. The vector polarization $P_{y}$ results from the vector polarized final deuteron along $y$ direction which is perpendicular to the scattering plane. In the Born (OPE) approximation with electron mass neglected, $P_{y}=\operatorname{Im}\left(T_{11}\right)=0$. The tensor (quadrupole) polarizations can be obtained from two ways. The first way is to use the unpolarized electron beam and the polarized deuteron target. The tensor polarizations are given by the ratio between the cross section with the definite polarizations of the deuteron target and the unpolarized cross section. Another way is to measure the polarization of the recoiled deuteron with the unpolarized beam and target [18]. A detailed discussion about the polarizations can be found in [22].

The polarization which has been most widely discussed is $P_{z z}\left(T_{20}\right)$. In the present general case, it is given as

$$
-I_{0} P_{z z}=\frac{8}{3} \tau\left(G_{C} G_{Q}\right)+\frac{8}{9} \tau^{2} G_{Q}^{2}+\frac{1}{3} \tau\left[1+2(1+\tau) \tan ^{2} \frac{\theta}{2}\right] G_{M}^{2}+\Delta P_{z z},
$$

where $\Delta P_{z z}=\delta P_{z z}+\delta_{0} P_{z z}$ with

$$
\begin{aligned}
\delta P_{z z}= & \frac{4 \tau^{2}}{3}\left[2(2 \tau+1) G_{1}-(4 \tau+1) G_{2}+4 \tau(\tau+1) G_{3}\right] \operatorname{Re}\left(G_{4}^{(2) *}\right) \\
& +\frac{4}{3} \tau \cot ^{2} \frac{\theta}{2}\left[\frac{4 \tau^{2}+2 \tau+1}{\tau+1} G_{1}-\frac{\tau(4 \tau+1)}{\tau+1} G_{2}+4 \tau^{2} G_{3}\right] \operatorname{Re}\left(G_{4}^{(2) *}\right) \\
& +\frac{2 K_{0}}{3 M}\left[\left(\frac{4 \tau^{2}+2 \tau+1}{\tau+1} G_{1}-\frac{3 \tau(2 \tau+1)}{2(\tau+1)} G_{2}+4 \tau^{2} G_{3}+2 \tau^{2} \tan ^{2} \frac{\theta}{2} G_{2}\right) \operatorname{Re}\left(G_{5}^{(2) *}\right)\right. \\
& \left.+\tau\left(4(2 \tau+1) G_{1}-(8 \tau+1) G_{2}+8 \tau(\tau+1) G_{3}\right) \operatorname{Re}\left(G_{6}^{(2) *}\right)\right]
\end{aligned}
$$

and

$$
\begin{aligned}
\delta_{0} P_{z z}= & \frac{8}{3} \tau\left[G_{C} \operatorname{Re}\left(G_{Q}^{(2) *}\right)+G_{Q} \operatorname{Re}\left(G_{C}^{(2) *}\right)\right] \\
& +\frac{16}{9} \tau^{2} G_{Q} \operatorname{Re}\left(G_{Q}^{(2) *}\right)+\frac{2}{3} \tau\left[1+2(1+\tau) \tan ^{2} \frac{\theta}{2}\right] G_{M} \operatorname{Re}\left(G_{M}^{(2) *}\right) .
\end{aligned}
$$

Note that $\delta_{0} P_{z z}$ is obtained from replacing $G_{i}$ by $\tilde{G}_{i}$ in the first three terms in Eq. (18) and retain only the OPE-TPE interference terms. It should be stressed that this polarization observable is often measured to determine the three from factors $G_{C, Q, M}$ in the literature 
[20, 21], because it relates to $G_{Q}^{2}$ and $G_{Q} G_{C}$.

The general form for the tensor polarization $P_{x z}$ (or $T_{21}$ ) is

$$
I_{0} P_{x z}=-\tau \frac{K_{0}}{M_{D}} \tan \frac{\theta}{2} G_{M} G_{Q}+\Delta P_{x z},
$$

where $\Delta P_{x z}=\delta P_{x z}+\delta_{0} P_{x z}$ with

$$
\begin{aligned}
\delta P_{x z}= & \tau\left\{-\frac{K_{0}}{M_{D}}\left[\tan \frac{\theta}{2} \tau G_{2}-\frac{\cot \frac{\theta}{2}}{\tau+1}\left(2 \tau G_{1}-(3 \tau+1) G_{2}+2 \tau(\tau+1) G_{3}\right)\right] \operatorname{Re}\left(G_{4}^{(2) *}\right)\right. \\
& +\tan \frac{\theta}{2}\left[\left(2 \tau G_{1}+(2 \tau-1) G_{2}+2 \tau(\tau+1) G_{3}\right) \operatorname{Re}\left(G_{5}^{(2) *}\right)-4 \tau(\tau+1) G_{2} \operatorname{Re}\left(G_{6}^{(2) *}\right)\right] \\
& +2 \tau \cot \frac{\theta}{2}\left[2\left(\frac{1}{\tau+1} G_{1}+G_{3}\right) \operatorname{Re}\left(G_{5}^{(2) *}\right)\right. \\
& \left.\left.+\left(G_{1}-4 G_{2}+2(\tau+1) G_{3}\right) \operatorname{Re}\left(G_{6}^{(2) *}\right)\right]\right\},
\end{aligned}
$$

and

$$
\delta_{0} P_{x z}=-\tau \frac{K_{0}}{M_{D}} \tan \frac{\theta}{2}\left[G_{M} \operatorname{Re}\left(G_{Q}^{(2) *}\right)+G_{Q} \operatorname{Re}\left(G_{M}^{(2) *}\right)\right] .
$$

For the tensor polarization $\left(P_{x x}-P_{y y}\right)$ (or $\left.T_{22}\right)$, we obtain

$$
I_{0}\left(P_{x x}-P_{y y}\right)=-\tau G_{M}^{2}+\Delta\left(P_{x x}-P_{y y}\right),
$$

where $\Delta\left(P_{x x}-P_{y y}\right)=\delta\left(P_{x x}-P_{y y}\right)+\delta_{0}\left(P_{x x}-P_{y y}\right)$ with

$$
\begin{aligned}
\delta\left(P_{x x}-P_{y y}\right)= & 4 \tau\left[\tau G_{2}+\frac{\cot ^{2} \frac{\theta}{2}}{\tau+1}\left(G_{1}+\tau G_{2}\right)\right] \operatorname{Re}\left(G_{4}^{(2) *}\right) \\
& +\frac{2 K_{0}}{M_{D}}\left[\left(\frac{1}{\tau+1} G_{1}+\frac{\tau}{\tau+1} G_{2}\right) \operatorname{Re}\left(G_{5}^{(2) *}\right)+\tau G_{2} \operatorname{Re}\left(G_{6}^{(2) *}\right)\right],
\end{aligned}
$$

and

$$
\delta_{0}\left(P_{x x}-P_{y y}\right)=-2 \tau G_{M} \operatorname{Re}\left(G_{M}^{(2) *}\right) .
$$

Since both the OPE and the TPE are included in the present calculation, we obtain non-vanishing polarization $P_{y}$. It is given by

$$
\begin{aligned}
I_{0} P_{y}= & \frac{2}{3} \tan \frac{\theta}{2}\left\{\frac { K _ { 0 } } { M _ { D } } \left[-(\tau+1)\left(G_{1} \operatorname{Im}\left(G_{2}^{(2) *}\right)+G_{2} \operatorname{Im}\left(G_{1}^{(2) *}\right)\right)\right.\right. \\
& -\tau(\tau+1)\left(G_{2} \operatorname{Im}\left(G_{3}^{(2) *}\right)+G_{3} \operatorname{Im}\left(G_{2}^{(2) *}\right)\right) \\
& \left.+\tau\left(\cot ^{2} \frac{\theta}{2}\left(2 G_{1}-G_{2}+2 \tau G_{3}\right)+\tau G_{2}\right) \operatorname{Im}\left(G_{4}^{(2) *}\right)\right] \\
& +\left[2 \tau \cot ^{2} \frac{\theta}{2}\left(2 G_{1}-G_{2}+2 \tau G_{3}\right)+\tau\left(2(\tau+1) G_{1}+G_{2}+2 \tau(\tau+1) G_{3}\right)\right] \operatorname{Im}\left(G_{5}^{(2) *}\right) \\
& \left.+4 \tau(\tau+1)\left[\cot ^{2} \frac{\theta}{2}\left(G_{1}+\tau G_{3}\right)+\tau G_{2}\right] \operatorname{Im}\left(G_{6}^{(2) *}\right)\right\} .
\end{aligned}
$$


The other two vector polarizations $P_{x}$ (or $\left.T_{11}\right) P_{z}$ (or $T_{10}$ ) do not vanish in the OPE approximation and have not been measured in the polarized electron deuteron scattering because they are too small to be practical. In the presence of TPE contribution, it can be written as

$$
I_{0} P_{z}=\frac{1}{3} \frac{K_{0}}{M_{D}} \sqrt{\tau(\tau+1)} \tan ^{2} \frac{\theta}{2} G_{M}^{2}+\Delta P_{z},
$$

where $\Delta P_{z}=\delta P_{z}+\delta_{0} P_{z}$ with

$$
\begin{aligned}
\delta P_{z}= & -\frac{2 \tau}{3} \sqrt{\frac{\tau}{\tau+1}}\left[\frac{K_{0}}{M_{D}} G_{2} \operatorname{Re}\left(G_{4}^{(2) *}\right)\right. \\
& \left.+\left(3+2(\tau+1) \tan ^{2} \frac{\theta}{2}\right) G_{2} \operatorname{Re}\left(G_{5}^{(2) *}\right)+2(\tau+1) G_{2} \operatorname{Re}\left(G_{6}^{(2) *}\right)\right],
\end{aligned}
$$

and

$$
\delta_{0} P_{z}=\frac{2}{3} \frac{K_{0}}{M_{D}} \sqrt{\tau(\tau+1)} \tan ^{2} \frac{\theta}{2} G_{M} \operatorname{Re}\left(G_{M}^{(2) *}\right)
$$

and we obtain the single polarization $P_{x}$

$$
I_{0} P_{x}=-\frac{4}{3} \sqrt{\tau(\tau+1)} \tan \frac{\theta}{2} G_{M}\left(G_{c}+\frac{1}{3} \tau G_{Q}\right)+\Delta P_{x}
$$

where $\Delta P_{x}=\delta P_{x}+\delta_{0} P_{x}$ with

$$
\begin{aligned}
\delta P_{x}= & \frac{1}{3} \sqrt{\tau(\tau+1)} \tan \frac{\theta}{2}\left\{-4 \tau^{2}\left(1+\frac{1}{\tau+1} \cot ^{2} \frac{\theta}{2}\right) G_{2} \operatorname{Re}\left(G_{4}^{(2) *}\right)\right. \\
& \left.+\frac{K_{0}}{M_{D}}\left[\left(2 G_{1}-\frac{4 \tau+1}{\tau+1} G_{2}+2 \tau G_{3}\right) \operatorname{Re}\left(G_{5}^{(2) *}\right)-4 \tau G_{2} \operatorname{Re}\left(G_{6}^{(2) *}\right)\right]\right\}
\end{aligned}
$$

and

$$
\begin{aligned}
\delta_{0} P_{x} & =-\frac{4}{3} \sqrt{\tau(\tau+1)} \tan \frac{\theta}{2}\left[G_{M}\left(\operatorname{Re}\left(G_{c}^{(2) *}\right)+\frac{1}{3} \tau \operatorname{Re}\left(G_{Q}^{(2) *}\right)\right)\right. \\
& \left.+\left(G_{c}+\frac{1}{3} \tau G_{Q}\right) \operatorname{Re}\left(G_{M}^{(2) *}\right)\right] .
\end{aligned}
$$

\section{Discussions}

The extractions of the deuteron form factors have so far been carried out within the OPE approximation. The motivation of investigating the $e D$ scattering beyond the OPE approximation is to identify the possible contribution of the TPE. The extractions of the deuteron form factors would have to be modified when the multiple-photon exchange effects become non-negligible. As a result it is important to know how and where the TPE effects will begin 
to play a role in the $e D$ scattering.

A simple and straightforward way to identify the TPE signature is to find some constraints between observables in the framework of the OPE approximation. Any deviation from these constraints would arise from the TPE effects. In particular, if we can find some $\theta$-independent combinations of physical observables then it will not be difficult to check them experimentally. We have found two such quantities, namely,

$$
\begin{aligned}
& \mathcal{C}_{1}=I_{0}\left(1+2 P_{z z}\right)=G_{C}^{2}-\frac{16}{3} \tau G_{C} G_{Q}-\frac{8}{9} \tau^{2} G_{Q}^{2}, \\
& \mathcal{C}_{2}=\frac{\left(I_{0} P_{x z}\right)\left(I_{0} P_{x}\right)}{I_{0} P_{z}}=4 \tau G_{Q}\left(G_{C}+\frac{\tau}{3} G_{Q}\right) .
\end{aligned}
$$

In Eqs. (34 35), the r.h.s. depend on $Q^{2}$ only. However, $I_{0}, P_{z z}, P_{x z}, P_{x}$, and $P_{z}$ on the l.h.s. depend both on $\theta$ and $Q^{2}$ but their $\theta$-dependence cancels out in these two particular combinations. Note that in Eqs. (34 35), we do not express $I_{0}$ in terms of $A$ and $B$ because such a separation holds only within the OPE framework. All the quantities which enter in Eqs. (34 [35) are directly measurable experimentally with no need of the separation between OPE and TPE contributions. Any $\theta$-dependence exhibited in $\mathcal{C}_{1}$ and $\mathcal{C}_{2}$ at any fixed value of $Q^{2}$ would be a clear indication of the two-photon exchange effects. In particular, should be possible to check Eq. (34) with the existing data. It will also serve as a good check for any theoretical prediction. In addition, it is easy to see that $\mathcal{C}_{1}+\frac{4}{3} \mathcal{C}_{2}=G_{c}^{2}+\frac{8}{9} \tau^{2} G_{Q}^{2}>0$.

Furthermore, it is also possible to derive some constraints at some specific angles. For example, when $\theta=\pi / 2$ one can easily derive three constraints as the following,

$$
\begin{aligned}
& P_{z}+\frac{2}{3} \sqrt{(\tau+1)(\tau+2)}\left(P_{x x}-P_{y y}\right)=0, \\
& (2 \tau+3) P_{z}^{2}-\frac{\tau}{6} \sqrt{(\tau+1)(\tau+2)} P_{x z} P_{x} \\
& +\frac{\tau^{2}}{6}(\tau+1) P_{x z}^{2}+\frac{3}{8}(\tau+2) P_{x}^{2}-\sqrt{(\tau+1)(\tau+2)} P_{z}=0, \\
& (2 \tau+3) P_{z}^{2}-\frac{4 \tau}{3} \sqrt{(\tau+1)(\tau+2)} P_{x z} P_{x}-2 \sqrt{(\tau+1)(\tau+2)} P_{z} P_{z z}=0 .
\end{aligned}
$$

Here all observables are measured at $\theta=\pi / 2$. Similar constraints at other angles such as $\theta=2 \pi / 3$ or $\theta=\pi / 3$ can also be found but with more complicated structure since $\tan (\theta / 2)=\cot (\theta / 2)=1$ when $\theta=\pi / 2$.

In the previous section, we have presented the most general forms for the differential cross section and polarizations in $e D$ elastic scattering. They are expressed in terms of six form 
factors which are the complex functions of $Q^{2}$ and $\epsilon$. We see that the TPE effects provide different $\theta$-dependence from the OPE in all observables. By analyzing the $\theta$-dependence of the kinematical pre-factors which appear with the interference terms of $G_{i} \tilde{G}_{j}^{(2) *}$ with $(i=1-3$, and $j=1-6)$, one is able to obtain some useful information about the TPE effects if $G_{i}^{(2)}$ are slowly varying functions of $\theta$. More specifically, these general expressions are greatly simplified at the forward and backward angles limitsaoc which enable us to disentangle the TPE effects from the OPE ones.

We first look at the unpolarized differential cross section. The differential cross section at a small scattering angle $\theta \sim 8^{0}$ has been parametrized in Ref. [13] as

$$
\frac{d \sigma}{d \Omega}=\left.\left(\sigma_{0} \cot ^{2} \frac{\theta}{2}\right)\right|_{\theta=8^{0}} \frac{a_{1}}{\left(1+q^{2} / a_{2}\right)^{a_{3}}}
$$

with parameters $a_{1,2,3}$. The $\cot ^{2}(\theta / 2)$-dependence of this parametrization derives from OPE, as seen in Eq. (17). In the small scattering angle region, say $\theta \leq 15^{0}, \cot ^{2}(\theta / 2) \geq 55>>1$, We have $\tan ^{2}(\theta / 2) \sim 1.9 \times 10^{-2} \sim 0$, and $K_{0} \sim 2 M_{D} \sqrt{\tau} \cot (\theta / 2)$. From Eq. (15), if terms with $\tan ^{2}(\theta / 2)$-dependence are neglected, we may then write,

$$
\begin{aligned}
\Delta \sigma \approx & \frac{a_{1}}{\left(1+q^{2} / a_{2}\right)^{a_{3}}}-\left[A\left(Q^{2}\right)+\Delta A\left(\theta, Q^{2}\right)\right] \\
= & \frac{1}{3}\left\{4 \tau \cot ^{2} \frac{\theta}{2}\left[(2 \tau-1) G_{1}-2 \tau G_{2}+2 \tau^{2} G_{3}\right] \operatorname{Re}\left(G_{4}^{(2) *}\right)\right. \\
& +4 \sqrt{\tau} \cot \frac{\theta}{2}\left[\left((2 \tau-1) G_{1}-2 \tau G_{2}+2 \tau^{2} G_{3}\right) \operatorname{Re}\left(G_{5}^{(2) *}\right)\right. \\
& \left.\left.+2 \tau\left((2 \tau+1) G_{1}-(2 \tau+1) G_{2}+2 \tau(\tau+1) G_{3}\right) \operatorname{Re}\left(G_{6}^{(2) *}\right)\right]\right\} .
\end{aligned}
$$

One thus finds that the TPE effects provide a much more complicated $\theta$-dependence for the differential cross section compared to Eqs. (51617) as obtained within the OPE approximation . In addition, that $\Delta \sigma$ should remain finite when $\theta$ approaches zero would require

$$
\operatorname{Re} G_{4}^{(2)}\left(\theta, Q^{2}\right) \leq \theta^{2}, \operatorname{Re} G_{5}^{(2)}\left(\theta, Q^{2}\right) \leq \theta, \quad \operatorname{Re} G_{6}^{(2)}\left(\theta, Q^{2}\right) \leq \theta
$$

when $\theta \rightarrow 0$.

Arguments similar to those presented above can also be applied to the polarization observables. At small angles, we obtain the following simplified results for the tensor polarizations

$$
\Delta P_{z z} \sim \frac{4}{3} \tau \cot ^{2} \frac{\theta}{2}\left[\frac{4 \tau^{2}+2 \tau+1}{\tau+1} G_{1}-\frac{\tau(4 \tau+1)}{\tau+1} G_{2}+4 \tau^{2} G_{3}\right] \operatorname{Re}\left(G_{4}^{(2) *}\right)
$$




$$
\begin{aligned}
& +\frac{4}{3} \sqrt{\tau} \cot \frac{\theta}{2}\left[\left(\frac{4 \tau^{2}+2 \tau+1}{\tau+1} G_{1}-\frac{3 \tau(2 \tau+1)}{2(\tau+1)} G_{2}+4 \tau^{2} G_{3}\right) \operatorname{Re}\left(G_{5}^{(2) *}\right)\right. \\
& \left.+\tau\left(4(2 \tau+1) G_{1}-(8 \tau+1) G_{2}+8 \tau(\tau+1) G_{3}\right) \operatorname{Re}\left(G_{6}^{(2) *}\right)\right] \\
\Delta P_{x z} \sim & \tau\left\{\left[2 \sqrt{\tau} \cot ^{2} \frac{\theta}{2}\left(\frac{2 \tau}{\tau+1} G_{1}-\frac{3 \tau+1}{\tau+1} G_{2}+2 \tau G_{3}\right)\right] \operatorname{Re}\left(G_{4}^{(2) *}\right)\right. \\
+ & 2 \tau \cot \frac{\theta}{2}\left[\left(\frac{1}{\tau+1} G_{1}+2 G_{3}\right) \operatorname{Re}\left(G_{5}^{(2) *}\right)\right. \\
& \left.\left.+\left(G_{1}-4 G_{2}+2(\tau+1) G_{3}\right) \operatorname{Re}\left(G_{6}^{(2) *}\right)\right]\right\} \\
\Delta\left(P_{x x}-P_{y y}\right) \sim & \frac{4 \tau}{\tau+1} \cot ^{2} \frac{\theta}{2}\left(G_{1}+\tau G_{2}\right) \operatorname{Re}\left(G_{4}^{(2) *}\right) \\
& +\frac{4 \sqrt{\tau}}{\tau+1} \cot ^{2} \frac{\theta}{2}\left[\left(G_{1}+\tau G_{2}\right) \operatorname{Re}\left(G_{5}^{(2) *}\right)+\tau(\tau+1) G_{2} \operatorname{Re}\left(G_{6}^{(2) *}\right)\right]
\end{aligned}
$$

We find that the TPE effects in $P_{z z}, P_{x z}$ and $P_{x x}-P_{y y}$ are similar to the ones in the differential cross section.

For vector polarizations $P_{x}$ and $P_{z}$, we obtain at the small angle limit

$$
\begin{aligned}
\Delta P_{z} \sim & -\frac{2 \tau}{3} \sqrt{\frac{\tau}{\tau+1}}\left[2 \sqrt{\tau} \cot \frac{\theta}{2} \operatorname{Re}\left(G_{4}^{(2) *}\right)+3 \operatorname{Re}\left(G_{5}^{(2) *}\right)+2(\tau+1) \operatorname{Re}\left(G_{6}^{(2) *}\right)\right] G_{2} . \\
\Delta P_{x} \sim & \frac{2 \tau \sqrt{\tau+1}}{3}\left[\left(2 G_{1}-\frac{4 \tau+1}{\tau+1} G_{2}+2 \tau G_{3}\right) \operatorname{Re}\left(G_{5}^{(2) *}\right)-4 \tau G_{2} \operatorname{Re}\left(G_{6}^{(2) *}\right)\right] \\
& -\frac{4}{3} \frac{\tau^{2} \sqrt{\tau}}{\sqrt{\tau+1}} \cot \frac{\theta}{2} G_{2} \operatorname{Re}\left(G_{4}^{(2) *}\right) .
\end{aligned}
$$

It is interesting to see the difference between $P_{x}, P_{z}$ and other observables. In the extreme forward limit, both $P_{x}$ and $P_{z}$ vanish while the other observables would remain finite. From Eqs. (41) and (45), one also sees that the TPE effects in $P_{x}$ and $P_{z}$ decrease faster than the corresponding effects in other observables which makes $P_{x}, P_{z}$ less interesting as far as the TPE effects are concerned.

In the large angle limit when the angle approaches $\pi$, i.e., very backward direction, expressions for the observables in $e D$ elastic scattering are also simplified if $G_{i}^{(2)}$ 's are slowly varying functions of $\theta$. However the Mott cross section is suppressed by $(\delta \theta)^{2}=(\pi-\theta)^{2}$, therefore no constraints such as Eq. (41) can be deduced. As long as $\operatorname{Re} G_{i}^{(2)}$ 's $(i=1-6)$ are analytical functions of $\delta \theta$, the observables are all finite when $\theta$ approach $\pi$. As a matter of fact, only the differential cross section, $P_{z z}$ and $P_{z}$ will receive nonvanishing TPE contribution in the backward directions. For example, $\theta \geq 165^{0}$, then $\cot ^{2}(\theta / 2) \leq 1.9 \times 10^{-2} \sim 0$ and $\tan ^{2}(\theta / 2)>55>>1$, such $x \sim 1$ and $K_{0} \simeq 2 M \sqrt{\tau(1+\tau)}$. For the unpolarized differential 
cross section in this limit, we then have

$$
\left.\frac{d \sigma}{d \Omega}\right|_{\theta \rightarrow \pi} \sim \sigma_{0}\left(B+\Delta B^{\prime}\right)
$$

with

$$
\Delta B^{\prime}=-\frac{8}{3} \tau \sqrt{\tau(1+\tau)} G_{2}\left(Q^{2}\right) \operatorname{Re}\left(G_{5}^{(2) *}\right)+\frac{8}{3} \tau(1+\tau) G_{2} \operatorname{Re}\left(G_{2}^{(2) *}\right) .
$$

For $P_{z z}\left(\right.$ or $\left.T_{20}\right)$ and $P_{z}$ (or $\left.T_{10}\right)$ one obtains

$$
\Delta P_{z z} \sim \frac{8}{3} \tau^{2} \sqrt{\tau(\tau+1)} \tan ^{2} \frac{\theta}{2} G_{2} \operatorname{Re}\left(G_{5}^{(2) *}\right)+\frac{4}{3} \tau(\tau+1) \tan ^{2} \frac{\theta}{2} G_{2} \operatorname{Re}\left(G_{2}^{(2) *}\right),
$$

and

$$
\Delta P_{z} \sim \frac{4}{3} \sqrt{\tau(\tau+1)} \tan ^{2} \frac{\theta}{2}\left[\sqrt{\tau(\tau+1)} \operatorname{Re}\left(G_{M}^{(2) *}\right)-\operatorname{Re}\left(G_{5}^{(2) *}\right)\right] G_{2}
$$

We note that the form factors which appear in Eqs. (47-49) are $\operatorname{Re}\left(G_{2}^{(2)}\right)$ and $\operatorname{Re}\left(G_{5}^{(2)}\right)$.

The vector polarization $P_{y}$ is unique in that it is related to the imaginary parts of the form factors. In the small angle limit, we may write

$$
\begin{aligned}
I_{0} P_{y} \sim & \frac{2}{3}\left\{2 \tau \sqrt{\tau} \cot ^{2} \frac{\theta}{2}\left[2 G_{1}-G_{2}+2 \tau G_{3}\right] \operatorname{Im}\left(G_{4}^{(2) *}\right)\right. \\
& +2 \tau \cot \frac{\theta}{2}\left[\left(2 G_{1}-G_{2}+2 \tau G_{3}\right) \operatorname{Im}\left(G_{5}^{(2) *}\right)\right. \\
& \left.\left.+2(\tau+1)\left(G_{1}+\tau G_{3}\right) \operatorname{Im}\left(G_{6}^{(2) *}\right)\right]\right\} .
\end{aligned}
$$

If $I_{0} P_{y}$ remain finite at small angles then the following conditions have to be satisfied:

$$
\operatorname{Im} G_{4}^{(2)}\left(\theta, Q^{2}\right) \leq \theta^{2}, \operatorname{Im} G_{5}^{(2)}\left(\theta, Q^{2}\right) \leq \theta, \operatorname{Im} G_{6}^{(2)}\left(\theta, Q^{2}\right) \leq \theta,
$$

when $\theta \rightarrow 0$. If Eq. (51) is satisfied then $P_{y}$ receives nonzero TPE effects at small angles. Since $P_{y}$ vanishes in OPE approximation, any measurement which yields a nonzero value of $P_{y}$ would be a manifestation of the TPE effects.

\section{Summary}

In summary, we considered the elastic $e D$ scattering beyond the one-photon-exchange approximation. The scattering amplitude is expressed in terms of six Lorentz structures as 
dictated by the charge-conjugation and parity invariance, together with helicity conservation. The six invariant functions $G_{i}$ 's, $i=1-6$ are complex functions of $Q^{2}$ and, e.g., $\theta$. We derived the general expressions for the complete set of seven observables, including the differential cross section, three vector polarizations and three tensor polarizations, in terms of the bilinear products $G_{i} G_{j}^{*}, i=1-3, j=1-6$, where $G_{j}=0$ for $j=4-6$ in the one-photon approximation. The general expressions which include the two-photon exchange effects were examined in details.

We found two $\theta$-independent relations as given in Eqs. (34) for any fixed value of $Q^{2}$ between these observables within the OPE approximation. Any deviation from these relations found in the experiments would be a clear indication of the two-photon-exchange mechanism. This is very useful since it will provide us with the much needed information about the precise kinematical region, i.e., values of momentum transfer square $Q^{2}$ and the scattering angle $\theta$, where the TPE effects would show up. In addition, we derived three relations in Eqs. (36+38), between polarization observables at $\theta=\pi / 2$. They also serve as useful criteria on the validity of the OPE approximation in the $e D$ scattering.

Finally, we discussed the possibilities of observing the TPE effects in some special kinematical regions, such as the forward and backward angles limit, under the assumption that the form factors $G_{i}$ 's are slowly varying functions of $\theta$. Whether such an assumption holds has to be substantiated by theoretical consideration. A model calculation in this direction is currently underway [23].

\section{Acknowledgements}

The work of Y.B.D. is supported in part by the National Sciences Foundations of China under grant No. 10475088. He would also like to thank Chinese Development Fund of Taiwan for support during his visit to the Department of Physics, National Taiwan University where he was warmly received. The work of C.W.K. is supported in part by the National Science Council of Taiwan under grant No. NSC95-2112-M033-014. The work of S.N.Y. and Y.C.C. is supported in part by the National Science Council of Taiwan under grant No. NSC952112-M002-025. We like to thank Haiqing Zhou for useful discussions. 


\section{References}

[1] J. Arrington, Phys. Rev. C 68, 034205 (2003).

[2] A. I. Akhiezer and M. P. Rekalo, Sov. J. Part. Nucl. 3, 277 (1974).

[3] M. K. Jones et al. [Jefferson Lab. Hall A Collaboration], Phys. Rev. Lett. 84, 1398 (2000).

[4] O. Gayou et al. [Jefferson Lab. Hall A Collaboration], Phys. Rev. Lett. 88, 092301 (2002).

[5] P. G. Blunden, W. Melnitchouk and J. A. Tjon, Phys. Rev. Lett. 91, 142304 (2003); S. Kondratyuk, P. G. Blunden, W. Melnitchouk, J. A. Tjon, Phys. Rev. Lett. 95, 172503 (2005).

[6] P. A. M. Guichon and M. Vanderhaeghen, Phys. Rev. Lett. 91, 142303 (2003).

[7] Y. C. Chen, A. Afanasev, S. J. Brodsky, C. E. Carlson and M. Vanderhaeghen, Phys. Rev. Lett. 93, 122301 (2004); A. Afanasev, S. J. Brodsky, C. E. Carlson, Y.C. Chen and M. Vanderhaeghen, Phys. Rev. D 72, 013008 (2005).

[8] P. G. Blunden, W. Melnitchouk, and J. A. Tjon, Phys. Rev. C 72, 034612 (2005).

[9] J. Gunion and L. Stodolsky, Phys. Rev. Lett. 30, 345 (1973)

[10] V. Franco, Phys. Rev. D 8, 826 (1973).

[11] V. N. Boitsov, L. A. Kondratyuk, and V. B. Kopeliovich, Sov. J. Nucl. Phys. 16, 238 (1973).

[12] F.M. Lev, Sov. J. Nucl. Phys. 21, 45 (1975).

[13] M. P. Rekalo and E. Tomasi-Gustafsson, Phys. Rev. C 60, 042202 (1999); M. P. Rekalo and E. Tomasi-Gustafsson, Nucl. Phys. A 740, 271 (2004).

[14] S. P. Wells et al. (SAMPLE), Phys. Rev. C 63, 064001 (2001).

[15] F.E. Maas et al., Phys. Rev. Lett. 94, 082001 (2005).

[16] V. Z. Jankus, Phys. Rev. 102, 1586 (1956); V. Glaser and B. Jaksic, Nuovo Cimento, 5, 1197 (1957). M. Gourdin, I. L. Nuovo Cimento, 28, 553 (1963) F. Gross, Phys. Rev. 142, 1025 (1966). 
[17] R. Tarrach, Nuovo Cimento A 28, 409 (1975).

[18] R. G. Arnold, C. E. Carlson, and F. Gross, Phys. Rev. C 21, 1426 (1980); Phys. Rev. C 23, 363 (1981).

[19] M. Garcon and J. W. Van Orden, Adv. Nucl. Phys. 26, 293 (2001).

[20] L. C. Alexa et al., [The Jefferson Lab. Hall A Collaboration], Phys. Rev. Lett. 82, 1374 (1999); D. Abbott, et al., [The Jefferson Lab $T_{20}$ Collaboration], Phys. Rev. Lett. 82, 1379 (1999); D. Abbott, et al., (The Jefferson Lab $T_{20}$ Collaboration), Phys. Rev. Lett. 84, 5053 (2000);

[21] M. Garcon et al., Phys. Rev. C 49, 2516 (1994); D. M. Nikolenko, Phys. Rev. Lett. 90, 072501 (2003), M. Bouwhuis et al., Phys. Rev. Lett. 82, 3755 (1999).

$[22]$ G. G. Ohlsen, Rep. Prog. Phys. 35, 717 (1972).

[23] H.Q. Zhou, Y.C. Chen, Y.B. Dong, C.W. Kao, and S.N. Yang, in preparation. 